\title{
REPRESENTAÇÃO E CONTROLE DE LAMINADORES TANDEM BASEADO EM FUNÇÕES DE SENSIBILIDADE OBTIDOS ATRAVÉS DE REDES NEURAIS
}

\author{
Luis E. Zárate*
}

Horacio Helman (i.m. $)^{\dagger}$

José M. Gálvez

*Laboratório LICAP, PUC-MG, Av. Dom José Gaspar 500, Coração Eucarístico, CEP. 30535-610, Belo Horizonte, MG, Brasil

${ }^{\dagger}$ Dept $^{o}$ de Engenharia Metalúrgica e de Materiais, EEUFMG

${ }^{\ddagger}$ Departamento de Engenharia Mecânica, EEUFMG

\begin{abstract}
The mathematical modeling of the tandem mills involves non lineal expressions of difficult analytic and numeric solutions. If the model proposed by Alexander (1972), considered the most complete (with larger effort computational) it be considered to describe the process in each stand, this hinders the application of that model in control and supervision systems "on-line". For resolve such difficulty, in this work a representation of the sensibility of the process by means of Neural networks is presented.

The new representation allows to obtain the value of the output thickness, for each stand, in the form predictive, eliminating the need of the sensor of thickness, usually ray-X, that introduce delays in the loops control.

Other aspect of this work is the application of predictive control for tandem mills, based in neural networks. Simulation results are presented.
\end{abstract}

KEYWORDS: Automobile Industry, Tandem mills, Automation, Predictive Control, Neural networks.

\footnotetext{
Artigo submetido em 20/12/00

1a. Revisão em 07/08/02

Aceito sob recomendação do Ed. Assoc. Prof. Fernando Gomide
}

\section{RESUMO}

A modelagem matemática do processo de laminação em tandem envolve expressões não lineares de difíceis soluções analíticas e/ou numéricas. Se o modelo proposto por Alexander (1972), considerado um dos mais completos e o de maior esforço computacional, for considerado para descrever o processo de deformação da tira em cada cadeira, isto dificultaria a aplicação deste modelo em sistemas de controle e supervisão "on-line". Para sobrelevar tal dificuldade, um dos propósitos deste trabalho é a representação da sensibilidade do processo por meio de Redes Neurais Artificiais.

A nova representação permite obter o valor da espessura final, de cada cadeira, na forma preditiva eliminando a necessidade de sensores de espessura, normalmente raio$\mathrm{X}$, que introduzem atrasos de tempos nas malhas de controle.

O segundo aspecto deste trabalho é a aplicação de controle preditivo para laminadores tandem com estruturas baseadas em redes neurais artificiais que permitam sobrelevar o retardo de tempo existente nestes processos. Resultados da representatividade do laminador tandem 
por meio de redes neurais e simulação do controlador proposto são apresentados.

PALAVRAS-CHAVE: Industria Automobilística, Laminação Tandem, Automação, Control Preditivo, Redes Neurais.

\section{INTRODUÇÃO}

Atualmente a industria automobilística utiliza de aços laminados à frio com dimensões que variam de $0,20 \mathrm{~mm}$ até $3,00 \mathrm{~mm}$ de espessura e de $700 \mathrm{~mm}$ até $1800 \mathrm{~mm}$ de largura. Uma forma de obter alta produtividade na obtenção desses produtos é utilizando processamento em tandem. O processo de laminação em tandem consiste na redução contínua da tira até a espessura final desejada, através das " $n$ "cadeiras do laminador (ver Figura 1). Este processo é conhecido pela alta produtividade podendo atingir velocidades de laminação da ordem de $1500 \mathrm{~m} / \mathrm{min}$. Nos últimos anos, os processos de laminação têm sido altamente automatizados embora nova tecnologia ainda é necessária para melhorar a qualidade dos produtos e aumentar a produção. A qualidade do produto está ligada à espessura e forma do material laminado, para alcançar esse objetivo aplicação de técnicas de Inteligência Artificial estão sendo utilizadas.

Técnicas de projeto de controladores têm sido propostas para solucionar o problema do controle de tiras laminadas a frio. Como exemplo pode-se citar: Hasegawa e Taki (1991), propuseram um sistema de controle de forma baseado em lógica nebulosa; em Hattori et al. (1992) foi apresentada uma estrutura baseada em lógica nebulosa e redes neurais para controle de planicidade; em Feldmann (1997) modelos matemáticos para simulação e controle de sistemas modernos de laminação foram discutidos e em Gonçalves e Helman (1998) foi proposto um controle de espessura de saída de tiras laminadas a frio utilizando lógica nebulosa.

Atualmente, o principal desafio no processo de laminação em tandem é adaptar rapidamente as cadeiras posteriores nos casos onde acontece algum tipo de perturbação nos parâmetros do processo, por exemplo na espessura de entrada da tira. Para melhor controle do processo é necessário a presença de sistemas inteligentes que irão monitorar e diagnosticar possíveis desvios dos pontos de operação, avaliando os efeitos das perturbações em cada cadeira do laminador. Para isto, é necessário considerar modelos do processo de alto desempenho computacional (Zárate 1998).

Um dos modelos mais completos da teoria da laminação é o de Alexander (1972) e o mais recentemente proposto, fundamentado no modelo anterior, é o de Freshwater (1995). Ambos modelos exigem grande esforço computacional o que impede seu uso em sistemas de controle e supervisão on-line. Atualmente Redes Neurais Artificiais recebem grande atenção devido a capacidade em resolver problemas não-lineares por aprendizado. Atualmente, as representações de processos por meio de redes neurais estão sendo usadas na extração de regras que descrevem o comportamento do processo. Estas regras podem alimentar a base de conhecimento que sustenta um sistema especialista.

Por outro lado o desenvolvimento de novos sistemas de controle de abertura (Automatic Gauge Control AGC) constitue um importante campo de pesquisa na industria da laminação do aço. Estes sistemas podem variar quanto a forma e complexidade para diferentes configurações do laminador. Um AGC pode utilizar informação da espessura de saída, da carga de laminação e da abertura dos cilindros para ser realimentada pelo sistema de controle e assegurar que a espessura de saída da tira em cada cadeira permaneça o mais constante possível. Normalmente a técnica que tem sido utilizada para medir desvios na espessura é o posicionamento de um sensor de raio-X após a região de deformação cilindromaterial, o que causa um retardo de tempo no sistema de realimentação e produz uma significante deterioração no desempenho do controlador. Sensores de raio-X têm custo alto, por esta razão em laminadores tandem são instalados unicamente na última cadeira do laminador. Isto degrada a qualidade do produto na espessura final, desde que as maiores perturbações encontram-se nas primeiras cadeiras do laminador.

Em Zárate et.al. (1998a) e Zárate et.al. (1999) foram apresentadas duas estruturas de controle de espessura para laminadores de uma única cadeira. A técnica apresentada neste trabalho, para controle de espessura de laminadores tandem, foi apresentada em Zárate (1998). Esta técnica não segue qualquer aproximação clássica e utiliza diretamente a medida da abertura para implementar um controlador baseado em modelo preditivo de espessura proposto em Zárate et.al. (1998b). Através do modelo preditivo é possível eliminar o retardo de tempo no laço de realimentação e eliminar os sensores de raio$\mathrm{X}$ substituindo-os por sensores virtuais localizados em cada cadeira do laminador tandem.

Este artigo é dividido em cinco seções: na seção 2, é apresentado o modelo do laminador tandem para " $n$ "cadeiras; na seção 3 é representado o processo através de redes neurais artificiais e discutido o modelo preditivo para a espessura de saída; na seção 4, é mostrado a estrutura do controlador e na seção 5 são apresentados 
resultados de simulação e as conclusões do trabalho.

\section{MODELO PARA A LAMINAÇÃO EM TANDEM}

Um laminador tandem é formado por " $n$ "cadeiras unidas pela tira que passa através delas. A tira é fornecida de uma bobina e quando laminada alimenta uma outra. A espessura da tira é reduzida em passes sequênciais através de uma alta pressão sobre uma pequena área de contato de 6,0-25,4 mm de comprimento. A força de compressão necessária é fornecida acima e abaixo dos cilindros de encosto pelos motores através de mecanismos hidráulicos ou de parafusos. Ver Figura 1.

Existem diversos modelos para calcular a carga necessária $(P)$ e o torque de laminação $(T q)$, os quais são funções não lineares de vários parâmetros: espessura inicial $\left(h_{i}\right)$, espessura final $\left(h_{f}\right)$, tensão à ré $\left(t_{r}\right)$, tensão à frente $\left(t_{f}\right)$, coeficiente de atrito $(\mu)$, tensão média de escoamento $(\bar{y})$, módulo de Young do material da tira $(E)$ e raio dos cilindros de trabalho $(R)$.

$$
\begin{aligned}
P & =f\left(\bar{y}, h_{i}, h_{f}, t_{r}, t_{f}, \mu, E, D\right) \\
T & =f\left(\bar{y}, h_{i}, h_{f}, t_{r}, t_{f}, \mu, E, D\right)
\end{aligned}
$$

Qualquer mudança na espessura de entrada, nas tensões à frente, à ré, na tensão de escoamento, no atrito ou na abertura dos cilindros, causará mudanças na carga e no torque de laminação e consequentemente na espessura de saída.

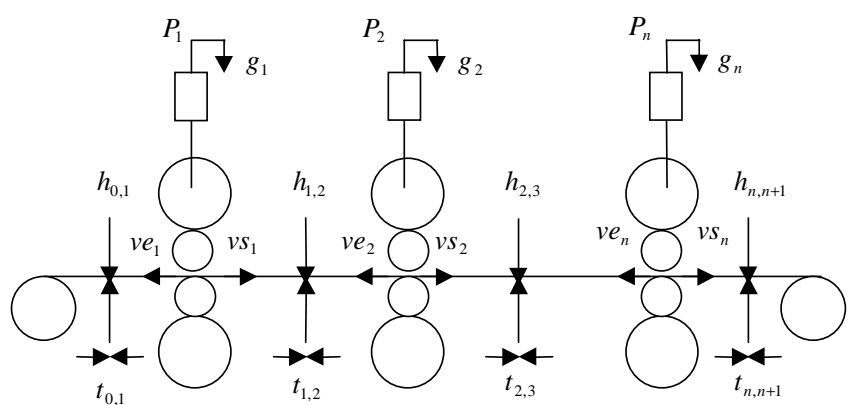

Figura 1: Esquema para um laminador tandem

Para obter um modelo do processo de laminação é necessário uma expressão para calcular a espessura de saída e a carga de laminação, quando perturbações ocorrem no processo. Observe na Eq. (1), que o valor da carga de laminação depende do conhecimento a priori do valor da espessura de saída e vice versa, existindo um "laço algébrico" na expressão que impede o cálculo analítico desses parâmetros simultaneamente. Em Zárate (2000) são discutidos, dois métodos de solução numérica e proposto um método iterativo de aproximações sucessivas para determinar a carga e a espessura final simultaneamente, quando perturbações ocorrem no processo. Embora o método proposto é válido para qualquer região de laminação, este apresenta características não desejadas em relação ao tempo computacional gasto. Uma outra forma para representar o processo de laminação é através dos fatores de sensibilidade, o qual permite obter uma expressão para cálculo da espessura de saída independente do conhecimento da carga de laminação, como visto a continuação.

As equações de sensibilidade para as Eq. (1) e Eq. (2) encontram-se dadas pelas Eq. (3) e Eq. (4) respectivamente.

$$
\begin{array}{r}
\Delta P=\frac{\partial P}{\partial h_{i}} \Delta h_{i}+\frac{\partial P}{\partial h_{f}} \Delta h_{f}+\frac{\partial P}{\partial t_{r}} \Delta t_{r}+\frac{\partial P}{\partial t_{f}} \Delta t_{f}+ \\
\frac{\partial P}{\partial \mu} \Delta \mu+\frac{\partial P}{\partial \bar{y}} \Delta \bar{y} \\
\Delta T_{q}=\frac{\partial T_{q}}{\partial h_{i}} \Delta h_{i}+\frac{\partial T_{q}}{\partial h_{f}} \Delta h_{f}+\frac{\partial T_{q}}{\partial t_{r}} \Delta t_{r}+\frac{\partial T_{q}}{\partial t_{f}} \Delta t_{f}+ \\
\frac{\partial T_{q}}{\partial \mu} \Delta \mu+\frac{\partial T_{q}}{\partial \bar{y}} \Delta \bar{y}
\end{array}
$$

Os termos das derivadas parciais envolvem expressões de difícil resolução. Em Denti (1994) foram obtidas expressões de sensibilidade para as expressões (1) e (2) utilizando o modelo de Orowan (1944). Em Denti (1994) é apresentada uma solução numérica para cada termo e obtido famílias de curva de sensibilidade de parâmetros. Para novos pontos de operação, novos cálculos numéricos são necessários. Expandindo as Eqs. (3) e (4) para as " $n$ "cadeiras do laminador, podem-se obter as Eqs. (5) e (6).

Qualquer variação na abertura dos cilindros, na cadeira "n", pode ser representada pela seguinte expressão:

$$
\Delta h_{n, n+1}=\Delta g_{n}+\frac{\Delta P_{n} W}{M_{n}}
$$

onde $W$ é a largura do material sendo laminado e $M$ o módulo de rigidez da cadeira de laminação.

Combinando as expressões (5) e (7) obtém-se a expressão (8) para cálculo da espessura de saída para as 


$$
\begin{aligned}
\Delta P_{1} & =\frac{\partial P_{1}}{\partial h_{0,1}} \Delta h_{0,1}+\frac{\partial P_{1}}{\partial h_{1,2}} \Delta h_{1,2}+\frac{\partial P_{1}}{\partial t_{0,1}} \Delta t_{0,1}+\frac{\partial P_{1}}{\partial t_{1,2}} \Delta t_{1,2}+\frac{\partial P_{1}}{\partial \mu_{1}} \Delta \mu_{1}+\frac{\partial P_{1}}{\partial y_{1}} \Delta y_{1} \\
\Delta P_{2} & =\frac{\partial P_{2}}{\partial h_{1,2}} \Delta h_{1,2}+\frac{\partial P_{2}}{\partial h_{2,3}} \Delta h_{2,3}+\frac{\partial P_{2}}{\partial t_{1,2}} \Delta t_{1,2}+\frac{\partial P_{2}}{\partial t_{2,3}} \Delta t_{2,3}+\frac{\partial P_{2}}{\partial \mu_{2}} \Delta \mu_{2}+\frac{\partial P_{2}}{\partial y_{2}} \Delta y_{2} \\
& \vdots \\
\Delta P_{n} & =\frac{\partial P_{n}}{\partial h_{n-1, n}} \Delta h_{n-1, n}+\frac{\partial P_{n}}{\partial h_{n, n+1}} \Delta h_{n, n+1}+\frac{\partial P_{n}}{\partial t_{n-1, n}} \Delta t_{n-1, n}+\frac{\partial P_{n}}{\partial t_{n, n+1}} \Delta t_{n, n+1}+\frac{\partial P_{n}}{\partial \mu_{n}} \Delta \mu_{n}+\frac{\partial P_{n}}{\partial y_{n}} \Delta y_{n} \\
\text { onde } & : \Delta P_{n}=P_{n}-P_{n}^{*} \\
\Delta T_{q 1} & =\frac{\partial T_{q 1}}{\partial h_{0,1}} \Delta h_{0,1}+\frac{\partial T_{q 1}}{\partial h_{1,2}} \Delta h_{1,2}+\frac{\partial T_{q 1}}{\partial t_{0,1}} \Delta t_{0,1}+\frac{\partial T_{q 1}}{\partial t_{1,2}} \Delta t_{1,2}+\frac{\partial T_{q 1}}{\partial \mu_{1}} \Delta \mu_{1}+\frac{\partial T_{q 1}}{\partial y_{1}} \Delta y_{1} \\
\Delta T_{q 2} & =\frac{\partial T_{q 2}}{\partial h_{1,2}} \Delta h_{1,2}+\frac{\partial T_{q 2}}{\partial h_{2,3}} \Delta h_{2,3}+\frac{\partial T_{q 2}}{\partial t_{1,2}} \Delta t_{1,2}+\frac{\partial T_{q 2}}{\partial t_{2,3}} \Delta t_{2,3}+\frac{\partial T_{q 2}}{\partial \mu_{2}} \Delta \mu_{2}+\frac{\partial T_{q 2}}{\partial y_{2}} \Delta y_{2} \\
& \vdots \\
\Delta T_{q n} & =\frac{\partial T_{q n}}{\partial h_{n-1, n}} \Delta h_{n-1, n}+\frac{\partial T_{q n}}{\partial h_{n, n+1}} \Delta h_{n, n+1}+\frac{\partial T_{q n}}{\partial t_{n-1, n}} \Delta t_{n-1, n}+\frac{\partial T_{q n}}{\partial t_{n, n+1}} \Delta t_{n, n+1}+\frac{\partial T_{q n}}{\partial \mu_{n}} \Delta \mu_{n}+\frac{\partial T_{q n}}{\partial y_{n}} \Delta y_{n} \\
\text { onde } & : \Delta T_{q n}=T_{q n}^{*}-T_{q n}
\end{aligned}
$$

"n"cadeiras do laminador tandem.

$\Delta h_{n, n+1}=\frac{1}{M_{n}-\frac{\partial P_{n}}{\partial h_{n, n+1}}}\left\{M_{n} \Delta g_{n}+\frac{\partial P_{n}}{\partial h_{n-1, n}} \Delta h_{n-1, n}+\right.$
$\left.\frac{\partial P_{n}}{\partial t_{n-1, n}} \Delta t_{n-1, n}+\frac{\partial P_{n}}{\partial t_{n, n+1}} \Delta t_{n, n+1}+\frac{\partial P_{n}}{\partial \mu_{n}} \Delta \mu_{n}+\frac{\partial P_{n}}{\partial y_{n}} \Delta y_{n}\right\}$

onde $\Delta h_{n, n+1}=h_{n, n+1}-h_{n, n+1}^{*}$ e $n=1, \ldots$, número de cadeiras. Onde $(*)$ representa o valor nominal de operação da variável correspondente. Onde os termos: $h_{0,1}, t_{0,1}$ correspondem a espessura inicial do material, sendo fornecido pelo sistema desbobinadeira e a tensão à ré da primeira cadeira respectivamente.

A Eq. (8) permite determinar o novo valor para a espessura de saída de cada cadeira quando ocorrem perturbações no laminador. Para resolver a Eq (8) é necessário a resolução dos termos derivativos ou fatores de sensibilidade. Esses termos dependem do modelo teórico considerado e são de grande complexidade para uma solução analítica e/ou numérica (Zárate 1998).

Em Zárate e Helman (2000) foi proposto um método numérico de aproximações sucessivas para encontrar o novo valor da espessura de saída e da carga de laminação quando ocorrem perturbações nos parâmetros. O método é aplicável para qualquer ponto de operação e embora elimine o cálculo dos fatores de sensibilidade, apresenta um tempo computacional elevado se comparado com a representação neural do processo de lamina- ção proposta neste trabalho (Zárate 1998c). Por outro lado os fatores de sensibilidade não serão obtidos diretamente de um modelo físico e sim a partir de uma rede neural artificial previamente treinada.

\section{REPRESENTAÇÃO DO PROCESSO DE LAMINAÇÃO POR MEIO DE REDES NEURAIS}

Nesta seção serão discutidos a representação do processo de laminação Eqs. (1) e (2) através de redes neurais e fatores de sensibilidade, Eq. (8), e a estrutura do modelo preditivo para o processo.

\subsection{Representação do Processo de Lamina- ção}

Nos últimos anos Redes Neurais Artificiais (RNA) estão sendo propostas como poderosas ferramentas computacionais devido aos baixos tempos de processamento que podem ser alcançados quando a rede está em operação. Estes tempos podem chegar a ordem de $460 \mu$ seg. (Zárate et al. $1998 \mathrm{c}$ ).

Para cada cadeira do laminador tandem, a representação neural do processo de laminação foi expressa através da relação Eq. (9), com seis entradas e duas saídas.

$$
\left(h_{i}, h_{f}, \mu, t_{r}, t_{f}, \bar{y}\right) \stackrel{\text { RedeNeural }}{\longrightarrow}\left(P, T_{q}\right)
$$


A RNA utilizada neste trabalho é a rede multicamadas, a qual se aproxima das teorias conexionistas que procuram descrever melhor o funcionamento do cérebro. O tipo de aprendizado desta rede é o aprendizado supervisionado, baseada no sistema "retropropagação do erro". A rede neural considerada é uma rede de duas camadas, onde o número de neurônios da camada escondida foi escolhido igual a $13(2 \mathrm{~N}+1$ neurônios, onde $\mathrm{N}$ é o número de entradas da rede, Kovács (1996)). O número de neurônios na camada de saída, igual a 2, correspondente ao número de saídas da rede. A função não linear sigmóide, foi escolhida neste trabalho como a função de transferência do axônio, por ser a mais consistente com a biofísica do neurônio biológico.

Para alimentar o processo de treinamento da RNA temse utilizado o modelo de Alexander (1972) como gerador do banco de dados. Geralmente o maior esforço para o treinamento de uma rede neural encontra-se na coleta e no pré-processamento dos dados. O pré-processamento consiste na normalização dos dados da entrada e da saída. Para o tipo de rede considerada o único requisito é que os valores das entradas e das saídas se encontrem no intervalo de 0 a 1.

Os seguintes procedimentos foram adotados para normalizar os dados das entradas associados às suas respectivas saídas, antes de usá-los no treinamento da rede neural:

a) Como os valores de 0 e 1 são valores infinitos para a função sigmóide, é recomendável diminuir este intervalo para valores de 0,2 e 0,8 respectivamente, com o objetivo de facilitar a convergência durante o treinamento da rede.

b) Os dados foram normalizados e desnormalizados através das seguintes expressões:

$$
\begin{aligned}
& f^{a}(L o)=L n=(L o-L m n) /(L \max -L \min ) \\
& f^{b}(L n)=L o=L n * L \max +(1-L n) * L m n
\end{aligned}
$$

onde : L $n$ é o valor normalizado; Lo o valor a normalizar; Lmín e Lmáx são valores mínimos e máximos dentre os valores reais das variáveis.

c) Pelo exposto no Item a) faze-se necessário a mudança de escala dos dados, para valores que proporcionem quando normalizados, valores limites entre 0,2 e 0,8 . As fórmulas para calcular Lmín e Lmáx são as seguintes:

$$
\begin{aligned}
& \text { Lmín }=(4 \times \text { LimiteInf. }- \text { LimiteSup }) / 3 \\
& \text { Lmáx }=(\text { LimiteInf. }-0,8 \times \text { Lmín }) / 0,2
\end{aligned}
$$

As equações (11a ) e (11b) são obtidas da substituição na equação (10 a) de $L n=0,2$ e Lo $=$ LimiteInf e de $L n=0,8$ e Lo $=$ LimiteSup. Onde LimiteInf e LimiteSup são os valores mínimos e máximos respectivamente dos conjuntos de dados originais.

\subsection{Representação do Processo por Meio dos Fatores de Sensibilidade}

O método para obter fatores de sensibilidade foi apresentado em Zárate (1998) e Zárate et.al. (1998b). O qual considera uma rede multicamadas, com uma camada escondida, com N: entradas, M: saídas e L:número de neurônios na camada escondida e a função sigmóide foi considerada como a função de transferência do axônio. A diferenciação da rede neural é genérica e depende unicamente dos valors $\mathrm{N}, \mathrm{M}, \mathrm{L}$ e dos pesos das camadas escondida e de saída obtidos durante o processo de treinamento da mesma.

A seguinte nomenclatura foi considerada para obtenção da expressão para cálculo dos fatores de sensibilidade através de redes neurais:

- $U_{i}, i=0, \ldots, N$ são as entradas originais (não normalizadas) da rede e $U_{0}=1$ é uma entrada de polarização

- $f_{i}^{a}() i=0,. \ldots, N$ são as funções normalizadoras das entradas (equação 10 a) com $f_{0}^{a}()=$.

- $X_{i}, i=0, \ldots, N$ são as entradas normalizadas com $X_{0}=U_{0}$

- $W_{i j}^{h}, i=1, \ldots, L$ e $j=0, \ldots, N$ contém o peso do neurônio i e entrada j para a camada escondida

- $n e t_{j}^{h}=\sum_{i=0}^{N} W_{j i}^{h} X_{i} j=1, \ldots, L$ produto de pesos pelas entradas

- $f_{j}^{h}\left(n e t_{j}^{h}\right) j=0, \ldots, L$ onde $f_{0}^{h}\left(n e t_{0}^{h}\right)=1$ é a função sigmóide para a camada escondida

- $I_{j}, j=0, \ldots, L$ são as respostas da função sigmóide $I_{0}=1$

- $W_{i j}^{o}, i=1, \ldots, M$ e $j=0, \ldots, L$ contém o peso do neurônio $i$ e entrada $j$, para a camada de saída

- $n e t_{j}^{o}=\sum_{i=0}^{L} W_{j i}^{o} I_{i}, j=1, \ldots, M$ produto de pesos pelas entradas, para a camada de saída

- $f_{j}^{o}\left(n e t_{j}^{h}\right), j=1, \ldots, M$ é a função sigmóide para a camada de saída 
- $Y_{j}, j=1, \ldots, M$ são as saídas normalizadas da rede, obtidas da função sigmóide

- $f_{i}^{b}(),. i=1, \ldots, M$ são as funções desnormalizadoras das saídas (ver equação 10 b)

- $Z_{i}, i=1, \ldots, M$ são as saídas originais da rede (desnormalizadas)

- $e \max _{k}$, e $\min _{k}, k=1, . ., N$ são as valores máximos e mínimos das entradas originais

- $s \max _{k}, s \min _{k}, k=1, . ., M$ são os valores máximos e mínimos das saídas originais

A continuação será apresentado o procedimento para obter as expressões de sensibilidade através de redes neurais artificiais (RNA). A saída real da rede em função da saída normalizada é representada pela expressão (12)

$$
\begin{gathered}
Z_{1}=f_{1}^{b}\left(Y_{1}\right) \\
Z_{2}=f_{2}^{b}\left(Y_{2}\right) \\
\vdots \\
Z_{M}=f_{M}^{b}\left(Y_{M}\right)
\end{gathered}
$$

Trabalhando adequadamente as variáveis, obtêm-se a equação (13) que relaciona as entradas com as saídas não normalizadas da RNA:

$$
\begin{gathered}
Z_{1}=f_{1}^{b}\left(f_{1}^{o}\left(\sum_{j=0}^{L} W_{1 j}^{o} f_{j}^{h}\left(\sum_{i=0}^{N} W_{j i}^{h} f_{i}^{a}\left(U_{i}\right)\right)\right)\right) \\
Z_{2}=f_{2}^{b}\left(f_{2}^{o}\left(\sum_{j=0}^{L} W_{2 j}^{o} f_{j}^{h}\left(\sum_{i=0}^{N} W_{j i}^{h} f_{i}^{a}\left(U_{i}\right)\right)\right)\right) \\
\vdots \\
Z_{M}=f_{M}^{b}\left(f_{M}^{o}\left(\sum_{j=0}^{L} W_{M j}^{o} f_{j}^{h}\left(\sum_{i=0}^{N} W_{j i}^{h} f_{i}^{a}\left(U_{i}\right)\right)\right)\right)
\end{gathered}
$$

Substituindo as expressões para as funções $f^{a}(),. f^{b}(),. f^{o}(),. f^{h}($.$) obtêm-se a equação (14)$

$$
\begin{aligned}
Z_{1} & =\frac{1}{1+\exp ^{-V_{1}}}\left[\operatorname{smax}_{1}-\operatorname{smin}_{1}\right]+\operatorname{smin}_{1} \\
Z_{2} & =\frac{1}{1+\exp ^{-V_{2}}}\left[\operatorname{smax}_{2}-\operatorname{smin}_{2}\right]+\operatorname{smin}_{2} \\
& \vdots \\
Z_{M} & =\frac{1}{1+\exp ^{-V_{M}}}\left[\operatorname{smax}_{M}-\operatorname{smin}_{M}\right]+\operatorname{smin}_{M}
\end{aligned}
$$

onde :

$$
V_{k}=\sum_{j=0}^{L} W_{k j}^{o} f_{j}^{h}\left(\sum_{i=0}^{N} W_{j i}^{h} f_{i}^{a}\left(U_{i}\right)\right), \text { para } k=1, \ldots, M
$$

Generalizando a equação (14), tem-se:

$$
Z_{k}=\frac{1}{1+\exp ^{-V_{k}}}\left[\operatorname{smax}_{k}-\operatorname{smin}_{k}\right]+\operatorname{smin}_{k}
$$

para $k=1, . ., M$. Os fatores de sensibilidade, serão dados pela equação (17)

$$
\frac{\partial Z}{\partial U}=\left[\begin{array}{cccc}
\frac{\partial Z_{1}}{\partial U_{1}} & \frac{\partial Z_{1}}{\partial U_{2}} & \cdots & \frac{\partial Z_{1}}{\partial U_{N}} \\
\cdots & \cdots & \cdots & \cdots \\
\frac{\partial Z_{M}}{\partial U_{1}} & \frac{\partial Z_{M}}{\partial U_{2}} & \cdots & \frac{\partial Z_{M}}{\partial U_{N}}
\end{array}\right]
$$

Cada termo da matriz de sensibilidade se calcula como:

$$
\frac{\partial Z_{k}}{\partial U_{i}}=\left[\operatorname{smax}_{k}-\operatorname{smin}_{k}\right] \frac{\exp ^{-V_{k}}}{\left(1+\exp ^{-V_{k}}\right)^{2}} \frac{\partial V_{k}}{\partial U_{i}}
$$

Trabalhando com o termo derivativo (da equação 18) e considerando a equação (15) tem-se:

$$
\frac{\partial V_{k}}{\partial U_{i}}=\frac{\partial}{\partial U_{i}}\left(W_{k 0}^{o}+\sum_{j=1}^{L} W_{k j}^{o} f_{j}^{h}\left(\sum_{i=0}^{N} W_{j i}^{h} f_{i}^{a}\left(U_{i}\right)\right)\right)
$$

Diferenciando a equação (19), introduzindo o resultado na equação (18), obtem-se a equação (20) que permite calcular os fatores de sensibilidade a partir da RNA

$$
\begin{gathered}
\frac{\partial Z_{k}}{\partial U_{i}}=\left[\begin{array}{cccc}
R_{1} W_{11}^{o} & R_{1} W_{12}^{o} & \cdots & R_{1} W_{1 L}^{o} \\
R_{2} W_{21}^{o} & R_{2} W_{22}^{o} & \cdots & R_{2} W_{2 L}^{o} \\
\vdots & \vdots & \ddots & \vdots \\
R_{M} W_{M 1}^{o} & R_{M} W_{M 2}^{o} & \cdots & R_{M} W_{M L}^{o}
\end{array}\right] \\
{\left[\begin{array}{cccc}
\frac{Q_{1} W_{11}^{h}}{\operatorname{emax}_{1}-e \min _{1}} & \frac{Q_{1} W_{12}^{h}}{\operatorname{emax}_{2}-e \min 2} & \cdots & \frac{Q_{1} W_{1 N}^{h}}{\operatorname{emax}_{2}-e \min W_{N}} \\
\frac{Q_{2} W_{22}^{h}}{\operatorname{emax}_{1}-e \min 1} & \frac{\cdots}{\operatorname{emax}_{2}-e \min 2} & \frac{Q_{2} W_{2 N}^{h}}{\operatorname{emax}_{N}-e \min } \\
\vdots & \vdots & \ddots & \vdots \\
\frac{Q_{L} W_{L 1}^{h}}{\operatorname{emax}_{1}-e \min _{1}} & \frac{Q_{L} W_{L 2}^{h}}{\operatorname{emax}_{2}-e \min _{2}} & \cdots & \frac{Q_{L} W_{L N}^{h}}{\operatorname{emax}_{N}-e \min _{N}}
\end{array}\right]}
\end{gathered}
$$

$$
\begin{gathered}
\operatorname{com}: Q_{k}=\frac{\exp ^{-\left(\sum_{i=0}^{N} W_{k i}^{h} X_{i}\right)}}{\left(1+\exp ^{-\left(\sum_{i=0}^{N} W_{k i}^{h} X_{i}\right)}\right)^{2}}, \quad k=1, \ldots, L \\
R_{k}=\left(\operatorname{smax}_{k}-\operatorname{smin}_{k}\right) \frac{\exp ^{-V_{k}}}{\left(1+\exp ^{-V_{k}}\right)^{2}}, \quad k=1, . ., M
\end{gathered}
$$

A Eq. (20) permite determinar os fatores de sensibilidade através da diferenciação da rede neural previamente treinada: 


\subsection{Modelo Preditivo para a Espessura de Saída}

Um sistema de controle de espessura para laminadores utiliza a espessura de saída como a variável realimentada. Usualmente o sensor de espessura é localizado a uma certa distância da região de contato cilindromaterial o que introduz um retardo de tempo no laço de controle realimentado e cujo valor depende da velocidade da tira a qual pode ser variante no tempo.

O retardo de tempo pode ser considerado constante se as tensões adjacentes a uma cadeira de laminação forem também constantes. Isto ocorre devido a mudanças nas tensões na tira causando as variações na velocidade da mesmo. Considerar o retardo de tempo constante é mais difícil de acontecer num laminador tandem pois as tensões dependem das variações ocorridas em cadeiras adjacentes. Assim, é de interesse um controlador que independa do retardo de tempo e de suas possíveis variações. A Eq. (8) apresenta uma forma preditiva para a espessura de saída, se os fatores de sensibilidade forem calculados a partir de redes neurais treinadas com conjuntos de dados obtidos na região de deformação cilindro-material. As equações do modelo preditivo para a espessura de saída são:

$$
\Delta h_{f}=K[S][\Delta u]
$$

como:

$$
\begin{gathered}
\Delta h_{n, n+1}=h_{n, n+1}-h_{n, n+1}^{*} \\
h_{f}=h_{f}^{*}-K[S][\Delta u]
\end{gathered}
$$

onde:

$$
\begin{aligned}
K & =\frac{W}{M-W \frac{\partial P}{\partial h_{f}}} \\
{[S] } & =\left[\frac{M}{W} \frac{\partial P}{\partial h_{i}} \frac{\partial P}{\partial t_{r}} \frac{\partial P}{\partial t_{f}} \frac{\partial P}{\partial \mu} \frac{\partial P}{\partial \bar{y}}\right] \\
{[\Delta u] } & =\left[\begin{array}{c}
\Delta g \\
\Delta h_{i} \\
\Delta t_{r} \\
\Delta t_{f} \\
\Delta \mu \\
\Delta \bar{y}
\end{array}\right]=\left[\begin{array}{c}
g^{*} \\
h_{i}^{*} \\
t_{r}^{*} \\
t_{f}^{*} \\
\mu^{*} \\
\bar{y}^{*}
\end{array}\right]-\left[\begin{array}{c}
g \\
h_{i} \\
t_{r} \\
t_{f} \\
\mu \\
\bar{y}
\end{array}\right]
\end{aligned}
$$

Neste trabalho as entradas de $[S]$ são obtidas pela diferenciação da saídas da rede neural que representa o processo de laminação.

\section{ESTRUTURA DO CONTROLADOR PROPOSTO PARA O LAMINADOR TANDEM}

Um sistema de controle automático de espessura AGC ("Automatic Gauge Control") tem por objetivo manter constante a espessura do produto laminado, num valor igual ao valor desejado, independente das perturbações que venham afetar o processo na região de contato cilindro-material ou "roll-gap".

A técnica proposta utiliza diretamente a medida da abertura do gap para implementar o controlador. Observe que a medição do gap elimina o retardo de tempo no laço de realimentação e em situações de espessuras não muito finas onde $g \approx h_{f}$ é possível atingir o propósito de controle.

Como o valor atual da espessura de saída $h_{f}$ não pode ser medido sem retardo de tempo utiliza-se o valor predito de $h_{f}$. Este valor é calculado utilizando a Eq. (22). Observe que o modelo preditivo está sendo implementado como um sensor virtual cuja saída é a espessura de saída predita.

A função de transferência da planta (motor de posicionamento do gap) para cada cadeira do laminador tandem foi escolhida como:

$$
\begin{aligned}
G_{p}(s) & =G_{\text {motor }}(s) \cdot G_{\text {gap }}(s)=\frac{w(s)}{E(s)} \cdot \frac{K_{E}}{S} \\
& =\frac{K_{m}}{T_{m} S+1} \cdot \frac{K_{E}}{S}=\frac{K_{m} K_{s}}{S\left(T_{m}+1\right)}
\end{aligned}
$$

onde $K_{m}$ : constante de ganho do motor; $K_{E}$ : constante de engrenagem (posição); $T_{m}$ : constante de tempo do motor

Um controlador para cada cadeira foi desenhado para ter as características proporcional+derivativa $(\mathrm{PD})$ :

$$
C(s)=K_{p}+K_{d} S
$$

\section{APLICAÇÃO E RESULTADOS}

Para obter os fatores de sensibilidade de $[S]$ para cada cadeira do laminador, foi escolhido o modelo de Alexander (1972) como o gerador do banco de dados para treinamento e posterior diferenciação das RNA.

Dados para simulação. Para este trabalho, foram considerados como entradas das redes neurais: a espessura de entrada da tira, a espessura de saída, o atrito, a tensão à frente, a tensão a ré e a tensão média de escoamento. Como saídas foram consideradas a carga $(P)$ e o torque $(T q)$ de laminação. A tabela 1 mostra os dados do 


$$
\begin{aligned}
& W^{h}=\left[\begin{array}{cccccc}
6.2010 & 9.4161 & -1.7278 & -0.2642 & 1.8475 & 1.3078 \\
-1.5239 & -9.2425 & -2.4238 & 0.0734 & 0.7813 & -6.0229 \\
-11.4365 & -1.7129 & 1.2562 & -0.2171 & -0.6941 & 3.3125 \\
-0.2767 & -8.3214 & -5.5920 & -0.2737 & -3.3595 & -0.8293 \\
3.3504 & 5.2707 & 3.4006 & 0.0343 & -0.5938 & 4.7363 \\
8.9832 & 3.9726 & 0.7990 & 9.7730 & 0.9096 & 5.5016 \\
-6.1621 & 3.4810 & 7.4542 & 0.5558 & -7.0447 & -0.9006 \\
-4.8199 & -3.2388 & 6.5208 & -3.4559 & -0.6086 & 8.9722 \\
0.8914 & -8.2071 & -7.6049 & -0.1518 & -2.2323 & -1.5593 \\
-1.2378 & -10.6099 & -0.0338 & 0.0399 & 0.4850 & -2.1458 \\
8.3913 & 2.3521 & 5.9967 & -8.1868 & -4.0553 & 1.8826 \\
-1.4947 & -11.9576 & -1.6808 & 0.0733 & 0.7387 & -4.2602 \\
2.9411 & 4.8319 & -11.5151 & -1.5484 & -4.0985 & -4.2330
\end{array}\right] \\
& W_{\text {bias }}^{h}=\left[\begin{array}{r}
-11.4784 \\
8.9265 \\
2.5654 \\
10.9804 \\
-10.4911 \\
-15.1094 \\
5.3887 \\
-4.5520 \\
7.1306 \\
8.8376 \\
-0.4583 \\
8.7774 \\
2.5081
\end{array}\right], \quad W^{o}=\left[\begin{array}{cc}
0.5503 & 0.4083 \\
-1.7551 & -2.1593 \\
-0.9245 & -0.1960 \\
-0.2976 & 0.2163 \\
4.2976 & 0.9087 \\
0.0021 & 0.0045 \\
0.1479 & 0.1406 \\
0.1378 & 0.0480 \\
-1.1542 & -0.0074 \\
-6.4508 & 1.0470 \\
-0.0276 & -0.0106 \\
-3.9372 & 2.4463 \\
0.1814 & 0.0746
\end{array}\right], W_{\text {bias }}^{o}\left[\begin{array}{r}
6.4599 \\
-1.4106
\end{array}\right] .
\end{aligned}
$$

\begin{tabular}{|c|c|c|c|c|c|}
\hline \multicolumn{6}{|c|}{$\mathrm{E}=20400 \mathrm{kgf} / \mathrm{mm}^{2} \quad \nu=0,330 \mathrm{R}=292,1 \mathrm{~mm}}$. \\
\hline & CADEIRA\#1 & CADEIRA\#2 & CADEIRA\#3 & CADEIRA\#4 & CADEIRA\#5 \\
\hline Espessura de Entrada (mm) : & 5,000 & 3,600 & 2,700 & 2,160 & 1,840 \\
\hline Espessura de Saída (mm) : & 3,600 & 2,700 & 2,160 & 1,840 & 1,700 \\
\hline Tensão à Ré (kgf/mm2) : & 0,441 & 9,098 & 9,393 & 9,765 & 10,230 \\
\hline Tensão à Frente (kgf/mm2) & 9,098 & 9,393 & 9,765 & 10,230 & 2,790 \\
\hline Coef de atrito : & 0,120 & 0,100 & 0,100 & 0,080 & 0,080 \\
\hline Tensão escoamto $(\mathrm{kgf} / \mathrm{mm} 2)$ : & 46,918 & 60,650 & 67,775 & 72,183 & 74,669 \\
\hline Redução do passe (\%) : & 28,0 & 25,0 & 20,0 & 14,8 & 7,6 \\
\hline Carga de Lamin. (kgf) : & 877,176 & 911,259 & 967,266 & 812.927 & 724.927 \\
\hline Torque (kgf-mm) : & 5171,757 & 5550,627 & 3937,503 & 2322,730 & 1927,288 \\
\hline GAP dos cilindros $(\mathrm{mm})$ : & 1,846 & 0,877 & 0,225 & 0,214 & 0,250 \\
\hline
\end{tabular}

Tabela 1: Dados do Material e de Operação do laminador

material e da operação das cadeiras para o laminador tandem.

Os valores da carga e torque utilizados para treinamento da rede foram obtidos através do modelo de Alexander (1972) considerando variações nas variáveis de entrada como sugerido em Bryant (1973): $\pm 3,0 \%$ para as espessuras de entrada e saída; $\pm 30,0$ para as tensões à frente e à ré; $\pm 20.0 \%$ para o atrito e $\pm 10,0 \%$ para a tensão média de escoamento. Para gerar os dados se considerou cada variável variando em três valores distintos.

Estrutura da Rede Neural. Foram consideradas redes neurais de duas camadas, onde o número de neurônios da camada escondida é igual a 13. O número de neurônios na camada de saída igual a 2, que corresponde ao número de saídas da rede,

Processo de Treinamento da Rede e Discusão dos

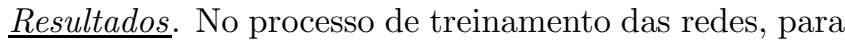
as cinco cadeiras de laminação, foram considerados pesos iniciais aleatórios, na faixa de $-1,0$ a $+1,0$. Os conjuntos de treinamento foram 729 conjuntos para cada cadeira, obtidos pela combinação das variações das variáveis do processo de laminação. A taxa de aprendizado foi de 0,008 para as cinco cadeiras do laminador. O erro médio quadrático para as cinco cadeiras foi de 0,040 e o número médio de iterações para treinamento foi de 540.000 .

Os pesos finais, para a primeira cadeira, das camadas escondida e de saída com seus pesos de polarização são 
Tabela 2: Fatores de Sensibilidade Obtidos da Diferenciação das RNA

\begin{tabular}{|c|c|c|c|c|c|c|}
\hline CADEIRA & $\frac{\partial P}{\partial h_{i}}$ & $\frac{\partial P}{\partial h_{f}}$ & $\frac{\partial P}{\partial \mu}$ & $\frac{\partial P}{\partial t_{r}}$ & $\frac{\partial P}{\partial t_{f}}$ & $\frac{\partial P}{\partial \bar{y}}$ \\
\hline$\# 1$ & 368,02 & $-2011,21$ & 1809,51 & $-40,40$ & $-0,15$ & 33,09 \\
\hline$\# 2$ & 929,64 & $-2034,84$ & 7762,37 & $-4,75$ & $-16,03$ & 45,34 \\
\hline$\# 3$ & 1644,75 & $-5122,16$ & 1425,37 & $-11,01$ & $-8,00$ & 27,07 \\
\hline$\# 4$ & 1777,45 & $-5603,19$ & 10289,22 & $-10,27$ & $-4,90$ & 13,83 \\
\hline$\# 5$ & 1859,43 & $-1380,79$ & 43160,83 & $-60,40$ & $-5,13$ & 12,38 \\
\hline
\end{tabular}

Tabela 3: Resultados Numéricos de Simulação

\begin{tabular}{|c|c|c|c|c|c|c|}
\hline \multicolumn{2}{|c|}{ Cadeira } & \multirow{2}{*}{$\# 1$} & \multirow{2}{*}{$\# 2$} & $\# 3$ & $\# 4$ & \multirow{2}{*}{$\# 5$} \\
\hline \multicolumn{2}{|c|}{ Perturbação em } & & & & & \\
\hline \multirow{2}{*}{$h_{i}$} & erro(\%) & 0,47 & 1,23 & 0,98 & 0,71 & 2,55 \\
\cline { 2 - 6 } & Tempo s-s & $6,67 \mathrm{~s}$. & $5,83 \mathrm{~s}$. & $8,33 \mathrm{~s}$. & 8,33 & $6,67 \mathrm{~s}$. \\
\hline \multirow{2}{*}{$t_{r}$} & erro (\%) & 0,04 & 0,06 & 0,10 & 0,07 & 1,57 \\
\cline { 2 - 7 } & Tempo s-s & $3,00 \mathrm{~s}$. & $1,67 \mathrm{~s}$. & $1,67 \mathrm{~s}$. & 5,00 & $6,67 \mathrm{~s}$. \\
\hline \multirow{2}{*}{$t_{f}$} & erro (\%) & 0,02 & 0,18 & 0,10 & 0,03 & 0,05 \\
\cline { 2 - 7 } & Tempo s-s & $1,67 \mathrm{~s}$. & $3,33 \mathrm{~s}$. & $3,33 \mathrm{~s}$. & 4,67 & $1,67 \mathrm{~s}$. \\
\hline \multirow{2}{*}{$\mu$} & erro (\%) & 0,06 & 0,23 & 0,09 & 0,20 & 2,55 \\
\cline { 2 - 6 } & Tempo s-s & $3,00 \mathrm{~s}$. & $5,00 \mathrm{~s}$. & $5,00 \mathrm{~s}$. & 6,67 & $6,67 \mathrm{~s}$. \\
\hline \multirow{2}{*}{$\bar{y}$} & erro (\%) & 0,38 & 0,99 & 0,41 & 0,25 & 0,69 \\
\cline { 2 - 6 } & Tempo s-s & $5,67 \mathrm{~s}$. & $4,67 \mathrm{~s}$. & $5,00 \mathrm{~s}$. & 8,33 & 4,17 \\
\hline
\end{tabular}

$$
\begin{aligned}
h_{f p} & =3,6-1,8054+\left[\begin{array}{llllllll}
0,33209 & 0,12221 & -0,01342 & -0,00005 & 0,60092 & 0,01099
\end{array}\right]\left[\begin{array}{llllll}
g & h_{i} & t_{r} & t_{f} & \mu & \bar{y}
\end{array}\right]^{T} \\
h_{f p} & =2,7-2,4898+\left[\begin{array}{lllllllll}
0,32951 & 0,30632 & -0,00156 & -0,00528 & 2,55775 & 0,01494
\end{array}\right]\left[\begin{array}{llllll}
g & h_{i} & t_{r} & t_{f} & \mu & \bar{y}
\end{array}\right]^{T} \\
h_{f p} & =2.16-1,0554+\left[\begin{array}{llllllll}
0,16334 & 0,26865 & -0,00180 & -0,00131 & 0,23282 & 0,00442
\end{array}\right]\left[\begin{array}{llllll}
g & h_{i} & t_{r} & t_{f} & \mu & \bar{y}
\end{array}\right]^{T} \\
h_{f p} & =1,84-0,8669+\left[\begin{array}{llllllll}
0,15144 & 0,26918 & -0,00156 & -0,00074 & 1,55821 & 0,00209
\end{array}\right]\left[\begin{array}{llllll}
g & h_{i} & t_{r} & t_{f} & \mu & \bar{y}
\end{array}\right]^{T} \\
h_{f p} & =1,7-3,1151+\left[\begin{array}{lllllll}
0,42003 & 0,78101 & -0,02537 & -0,00215 & 18,12876 & 0,00520
\end{array}\right]\left[\begin{array}{lllll}
g & h_{f} & \mu & \bar{y}
\end{array}\right]^{T}
\end{aligned}
$$

dados em (28).

Os pesos da RNAs podem ser substituídos na expressão (20) para obter os fatores de sensibilidade para os dados treinados. A tabela 2 mostra os fatores de sensibilidade de $[S]$, para as cinco cadeiras do laminador tandem.

Considerando a Eq. (22) e os valores nominais de operação das cinco cadeiras (tabelas 1 e 2), as Eqs. (29) mostram os preditores para cada cadeira.

As constantes dos controladores, Eq. (27) foram ajustados como: $K_{p}=8,00$ e $K_{D}=8$, 00 e foi observado que foram satisfatórios para todas as cadeiras facilitando a operação do laminador. Para analisar o desempenho do controlador foi simulado o seguinte caso. Perturbações:

$$
h_{i}(t)=\left\{\begin{array}{cc}
h_{i}^{*} & t<15 \\
1,03 * h_{i}^{*} & 15 \leq t \leq 30 \\
h_{i}^{*} & t>30
\end{array}\right.
$$

$$
\begin{aligned}
& t_{r}(t)=\left\{\begin{array}{cc}
t_{r}^{*} & t<45 \\
1,10 * t_{r}^{*} & 45 \leq t \leq 60 \\
t_{r}^{*} & t>60
\end{array}\right. \\
& t_{f}(t)=\left\{\begin{array}{cc}
t_{f}^{*} & t<75 \\
1,10 * t_{f}^{*} & 75 \leq t \leq 90 \\
t_{f}^{*} & t>90
\end{array}\right. \\
& \mu(t)=\left\{\begin{array}{cc}
\mu^{*} & t<105 \\
1,03 * \mu^{*} & 105 \leq t \leq 120 \\
\mu^{*} & t>120
\end{array}\right. \\
& \bar{y}(t)=\left\{\begin{array}{cc}
\bar{y}^{*} & t<135 \\
1,03 * \bar{y}^{*} & 135 \leq t \leq 150 \\
\bar{y}^{*} & t>150
\end{array}\right.
\end{aligned}
$$

As simulações são mostradas nas Figuras 2, 3 e 4 . A tabela 3 mostra os erros na espessura de saída obtidos das simulação. Observe-se que os maiores erros ocorrem com as perturbações na espessura de entrada, no atrito e na tensão de escoamento. O maior erro de 2,55\% acontece para as perturbações na espessura de entrada e no 
atrito para a última cadeira. Por tanto maior cuidado deve-se ter com a variação nesses parâmetros. Os maiores e menores tempo para alcançar o estado estacionário são $1.67 \mathrm{~s}$ e $8.33 \mathrm{~s}$ respectivamente.
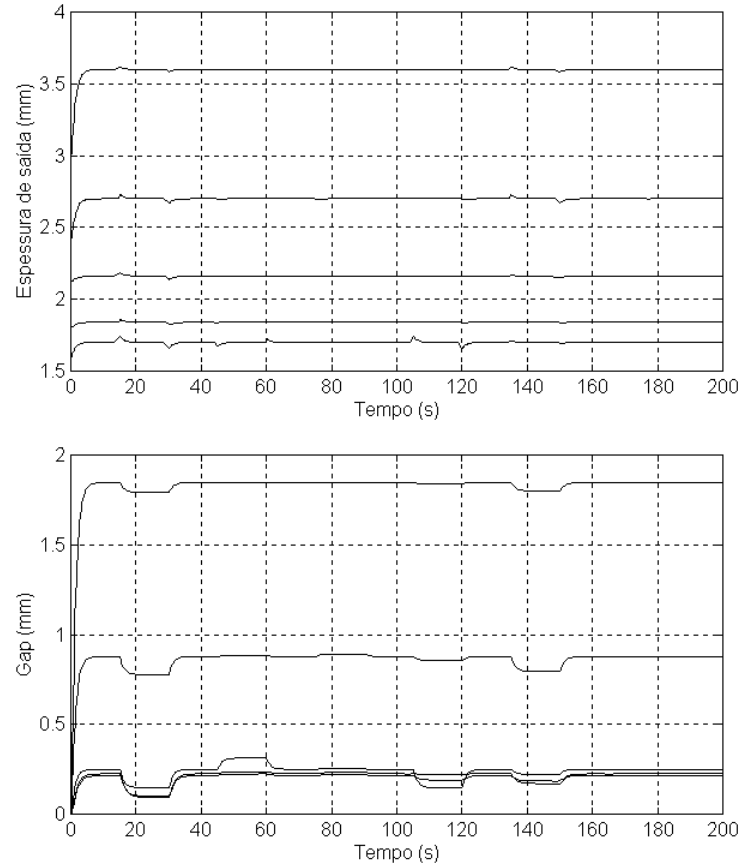

Figura 2: Respostas do Controlador a Perturbações nos Parâmetros

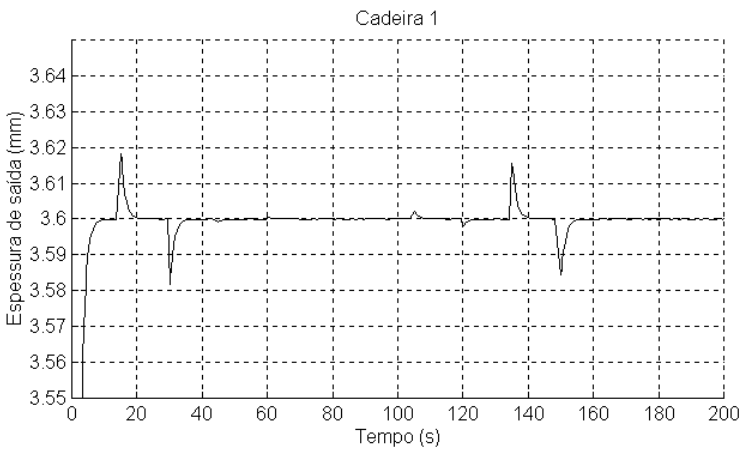

Figura 3: Zoom para as Resposta do Controlador em cada cadeira.

\section{CONCLUSÕES}

Neste artigo foi apresentado um método para controle de espessura para laminadores tandem. A estrutura do controlador proposto utiliza um modelo preditivo baseado em fatores de sensibilidade obtidos através da diferenciação de redes neurais previamente treinadas. Esta estrutura permite sobrelevar os atrasos de tempo existentes na dinâmica do sistema.
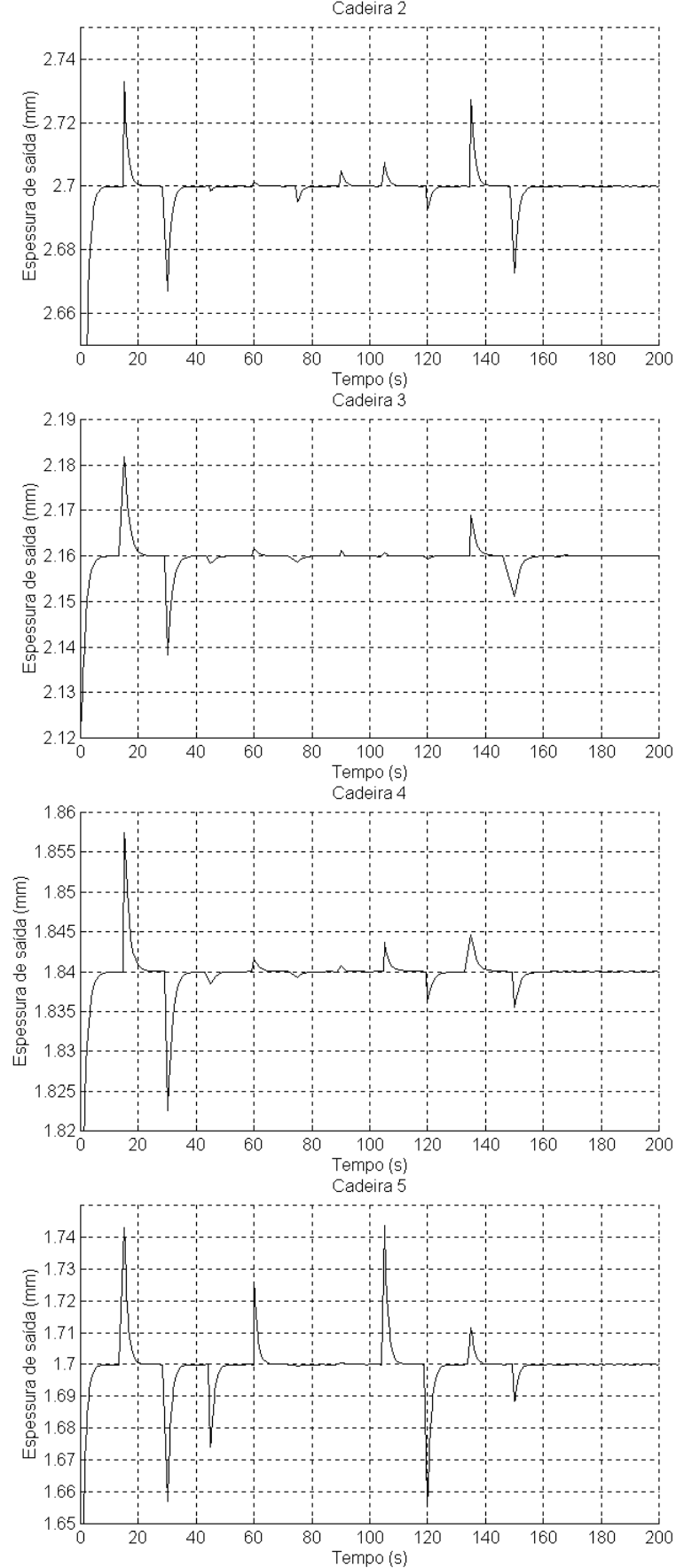

Figura 4: Zoom para as Resposta do Controlador em cada cadeira (continuação).

O modelo preditivo consiste na representação, através de redes neurais, do processo de deformação da tira na região de contato cilindro-material. Através da rede é determinado o valor da espessura no plano de saída do arco de contato, eliminando a necessidade do sensor de raio-X localizado a certa distância do plano. O modelo 
neural atua como um sensor virtual implementado por software.

Para garantir a exatidão do valor predito da espessura de saída, é necessário treinar a rede até alcançar um erro mínimo. Para o nosso caso o erro médio quadrático aceitável foi de 0,040 para todas as cadeiras. Uma limitação do modelo preditivo é o novo treinamento necessário se novos pontos de operação forem ajustados para uma cadeira. Isto pode ser solucionado com maior representatividade neural do processo de laminação.

A técnica foi aplicada num processo de laminação a frio e foi observado através de simulação que a resposta de tais sistemas pode ser melhorado por meio do uso de controladores preditivos. A análise dos resultados mostram que as respostas obtidas pelo controlador são aceitáveis para o processo de laminação. Embora o modelo preditivo, utilize fatores de sensibilidade calculados nas proximidades de um ponto de operação. Foi observado que para as perturbações o controlador ainda possui um comportamento satisfatório.

\section{REFERÊNCIAS}

Alexander, J.M., (1972). On the theory of Rolling. Proc. R. Soc. Lond. A. 326, pp. 535-563.

Byant, G.F., W.J. Edwards and C.H. McClure (1973). In G.F. Bryant (Ed.), Automation of Tandem Mills. pp. 6-6, The Iron and Steel Institute, London.

Denti, J. (1994). Um método de Controle Dinâmico de Laminadores Reversíveis. Tese de Doutoramento, EEUFMG, Belo Horizonte, MG, Brasil.

Feldmann, F., (1997), Mathematical models for simulation and control in flat rolling mills, MPT International, pp. 90-97.

Freshwater, I.J., (1995). Simplified Theories of Flat Rolling - I. The Calculation of Roll Pressure, Roll Force and Roll Torque, Int. J. Mech Sci. V. 18, n 6, pp. 633-648.

Gonçalves, R. \& Helman, H., (1998). Controle da Espessura de Saída de Tiras Laminadas Utilizando a Lógica Nebulosa, RBCM- J. of the Braz. Coc. Mechanical Sciences, V.XX - n. 4, pp. 645-655.

Hasegawa, A. and Taki, F., (1991). Development of Fuzzy Set Ttheory-based Shape control system for cold strip mill. Nippon Steel, Tokyo, n 49, pp. 59-62.

Hattori, S., Nakajima and M., Katayama, Y., (1992), Fuzzy Control Algorithm and Neural Networks for flatness control of a cold rolling process, Hitachi Review, V. 41, n. 1, pp. 31-38.

Kovács, Z.L. (1996). Redes Neurais Artificiais, Edição Acadêmica São Paulo, pp.75-76, São Paulo, Brasil.

Orowan, E. (1944). The Calculation of Roll Pressure in Hot and Cold Rolling. Proc. Inst. Mech. Eng., Vol. 150, pp. 140-167.

Zárate, L.E., (1998). Um Método para Análise da Laminação Tandem a Frio. Tese de Doutoramento, EEUFMG, Belo Horizonte, MG, Brasil.

Zárate, L.E., J.M. Gálvez and H. Helman, (1998a). A Neural Network Based Controller for Steel Rolling Mills Using Sensitivity Functions, Anais do $12^{\circ}$ Congresso Brasileiro de Automática, Uberaba M.G, Brasil, pp. 29-34.

Zárate, L.E., J.M. Gálvez and H. Helman, (1999). A Screw Control System for Rolling Mills Using Neural Networks, $15^{\text {th }}$ International Conference on CAD/CAM, Robotics and Factories of the Future, Águas de Lindóia S.P., Brasil, pp. RT2-6 - RT2-11.

Zárate, L.E., H. Helman, e J.M. Gálvez (1998b). Um Modelo para Processos Baseados em Fatores de Sensibilidade, Utilizando Redes Neurais, Anais do $12^{\circ}$ Congresso Brasileiro de Automática, Uberaba M.G, Brasil, pp.23-28.

Zárate, L.E., M. Song e H. Helman, (1998 c). Algoritmo para Linearização de Processos via Redes Neurais e sua Análise de Comportamento Assintótico. Anais do 5 Simpósio Brasileiro de Redes Neurais, Belo Horizonte, pp.199-204.

Zárate, L.E. e H. Helman, (1999a). Determination of the Thickness Control Parameters of the Rolling Process Through the Sensitivity Method, Using Neural Networks. The second International Conference on Intelligent Processing and Manufacturing of Materialss, Big Island, Hawaii, pp. 537-542.

Zárate, L.E., H. Helman, e J.M. Gálvez (1999b). A Method for the Determination of the Best Thickness Control Action in the Rolling Process, Through the Sensitivity Method, Using Neural Networks, $15^{\text {th }}$ International Conference on $C A D / C A M$, Robotics and Factories of the Futur, Águas de Lindóia S.P., pp. RT2-1 - RT2-5.

Zárate, L.E. e H. Helman, (2000). Um Modelo para Simulação da Laminação a Frio e Proposta de um Método para a Solução Numérica, Anais do $13^{\circ}$ Congresso Brasileiro de Automática, Florianópolis, S.C., pp. 1307-1313. 